Jens Kuhle

Raija L.P. Lindberg

Axel Regeniter

Matthias Mehling

Francine Hoffmann

Markus Reindl

Thomas Berger

Ernst W. Radue

David Leppert

Ludwig Kappos

\title{
Antimyelin antibodies in clinically isolated syndromes correlate with inflammation in MRI and CSF
}

Abstract Objective We investigated the correlation of antimyelin oligodendrocyte glycoprotein- (anti-MOG) and anti-myelin basic protein antibodies (antiMBP) in serum of CIS patients with inflammatory signs in MRI and in CSF and, as previously suggested, the incidence of more frequent and rapid progression to clinically definite MS (CDMS). Methods 133 CIS patients were analysed for anti-MOG and anti-MBP (Western blot). Routine CSF and cranial MRI (quantitatively and qualitatively) measures were analyzed. 55 patients had a follow-up of at least 12 months or until conversion to CDMS. Results Patients with antiMOG and anti-MBP had an increased intrathecal IgG production and CSF white blood cell count $(\mathrm{p}=0.048$ and $\mathrm{p}=0.036)$. When

D. Leppert, $\mathrm{MD}$

Biomarker and Experimental Medicine Units

Addenbroke's Centre for Clinical Investigation, GlaxoSmithKline R \& D Cambridge, Great Britain

L. Kappos, MD ( $\square)$

Head Outpatient Clinic Neurology and Neurosurgery

University Hospital Basel

Petersgraben 4

4031 Basel, Switzerland

Tel.: +41-61/265-4464

Fax: +41-61/265-5551

E-Mail: lkappos@uhbs.ch
anti-MBP alone, or both antibodies were present the cranial MRI showed significantly more T2 lesions $(\mathrm{p}=0.007$ and $\mathrm{p}=0.01$, respectively). There was a trend for more lesion dissemination in antiMBP positive patients $(p=0.076)$. Conversely, anti-MOG- and/or anti-MBP failed to predict conversion to CDMS in our follow-up group $(n=55)$. Only in female patients with at least one MRI lesion $(n=34)$ did the presence of anti-MOG correlate with more frequent $(\mathrm{p}=0.028)$ and more rapid $(\mathrm{p}=0.0209)$ transition to CDMS. Conclusions Presence of anti-MOG or anti-MBP or both was not significantly associated with conversion to CDMS in our CIS cohort. However, patients with anti-MOG and anti-MBP had higher lesion load and more disseminated lesions in cranial MRI as well as higher values for CSF leucocytes and intrathecal IgG production. Our data support a correlation of anti-MOG and antiMBP to inflammatory signs in MRI and CSF. The prognostic value of these antibodies for CDMS, however, seems to be less pronounced than previously reported.

Key words clinically isolated syndrome - prognosis · multiple sclerosis . antimyelin antibodies 


\section{Introduction}

Approximately $90 \%$ of relapsing remitting multiple sclerosis (MS) patients present with a clinically isolated syndrome (CIS) as the initial feature of their disease. Of these about $30-50 \%$ have symptoms and signs referable to multi-focal disease (polysymptomatic) $[43,45]$ and $50-80 \%$ are found to have clinically nonapparent cerebral white-matter lesions [30, 34]. Prognostic markers that allow for more accurate information about the patients' individual risk of developing MS are important, as not all patients will have a second episode defining conversion to clinically definite MS (CDMS). Accurate prognosis would influence treatment, because three randomized trials have shown that the development of CDMS is delayed by early interferon beta therapy [9, 19a].

Lesion load in the initial MRI of CIS patients has been up to now the best validated prognostic paraclinical measure $[6,24]$. However, neither clinical nor paraclinical markers (biofluid markers, MRI) have up to now allowed the prediction of the course of MS in an individual patient $[1,16]$. In a recent report patients with serum anti-MOG antibodies (anti-MOG) and anti-MBP antibodies (anti-MBP), determined by Western blot, developed CDMS more often and earlier than dual antibody-negative patients, suggesting that the measurement of these antibodies is a useful tool for predicting early conversion to CDMS [4].

There are several lines of evidence supporting a role of antibody-mediated demyelination in MS [29, 41]. Myelin oligodendrocyte glycoprotein (MOG) is a minor myelin component $(0.01-0.05 \%$ of central myelin protein) exclusively found in the CNS $[28,35]$. It consists of a 122 amino acid (aa) glycosylated n-terminal extracellular stretch, followed by a transmembranous (aa 123-150) and intracellular domain (aa 151-218). The n-terminal domain is expressed on the myelin surface, easily accessible to antibodies. Besides inducing encephalitogenic T-cells, anti-MOG lead to intensive demyelination in $\operatorname{EAE}[26,38,44]$. Anti-MOG are capable of inducing demyelination in brain cell cultures and have been detected in disrupted myelin of MS patients [17, 21].

Myelin Basic Protein (MBP) constitutes about 30\% of the central myelin protein and is localised in cytoplasm [3]. It is an extensively investigated autoantigen. The presence of antibodies against MBP in early MS is well established but their pathogenicity or relation to disease severity is still controversial $[11,37]$.

Using identical methods for antibody detection as Berger et al. [4], we investigated an independent cohort of patients with CIS. We are reporting on the relation of antibodies against myelin antigens with MRI and CSF measures and conversion to CDMS.

\section{Material and Methods}

\section{Patients}

To evaluate interlaboratory reproducibility prior to this study 39 sera of MS patients were analyzed for anti-MOG and anti-MBP independently in Innsbruck (M. Reindl and T. Berger) and in our laboratory using identical methods.

Subsequently we enrolled 60 CIS patients seen in our department from 1998 to 2003 (Figure 1). The diagnosis of CIS was based on the acute or subacute occurrence of a first clinical event suggestive of MS lasting for at least 24 hours. Fatigue alone and transient fever-related worsening of symptoms were not considered. Patients who had a history of any kind of previous neurological symptoms or clinical, laboratory, MRI, or CSF findings suggestive of any diagnosis other than MS were excluded. Patients were followed by visits at 6 or 12 month intervals including a neurological examination and assessment for relapses to document the interval between first clinical symptoms and CDMS. A diagnosis of CDMS was established when new symptoms or exacerbation of a previously stable or improving deficit at least four weeks after the initial event persisting for more than 24 hours occurred. Minimal follow-up was twelve months or the time point of CDMS diagnosis (mean follow-up 32.9 months, SD: 19.9 months, range 12-77.2). $5 / 60$ patients had no follow-up visit (four patients were lost to follow-up and one refused a follow-up visit). Sera were drawn at the earliest timepoint after initial presentation, stored at $-80^{\circ} \mathrm{C}$ and examined retrospectively. The initial MRI of these patients was also evaluated retrospectively.

We included additionally 73 patients from a prospective national Swiss project which offers practicing neurologists the evaluation of anti-MOG and anti-MBP status of their CIS patients (Figure 1). Participating neurologists provided clinical information in a standardized questionnaire.

\section{Image analysis}

Hard copies of the MRI scans were re-evaluated centrally. High quality and fully documented T2-, PD-weighted, FLAIR plane and gadolinium-enhanced T1-weighted scans with $5 \mathrm{~mm}$ slices performed at 1.5 or $3 \mathrm{~T}$ were available for 109 of 133 patients. Two experienced neuroradiologists, without access to the clinical information or antibody status, assessed the scan quality and eventually recorded the number and localization of detected abnormalities. High signal lesions were counted per slice in T2-

A. 39 MS patients

- interlaboratory reproducibility
B. 60 CISpatients

- predictive value

- cross sectional comparison of

antibody results, clinical and MR

findings
C. 73 CIS patients

- cross sectional comparison of antibody results, clinical and MR findings
Fig. 1 Description of study populations. Prior to analyzing the CIS patient sera $(n=133)$ for anti-MOG and anti-MBP 39 MS patient sera were analyzed for interlaboratory reproducibility of the assay (A). The core study included 60 CIS patients seen initially between 1998-2003 in our department and followed at 6 or 12 month intervals (B). In addition baseline findings of 73 CIS patients from a prospective national Swiss project were correlated with clinical and MRI characteristics (C) 
weighted SE and FLAIR images and categorized according to four prespecified regions of the brain: 1 . cortical/subcortical; 2 . Corpus callosum; 3. periventricular; 4 . posterior cranial fossa.

Contrast enhancing lesions and black holes (low-signal lesions) were also counted per slice on the enhanced T1-weighted images.

\section{Western blot analysis for anti-MOG and anti-MBP antibodies}

The human recombinant extracellular MOG domain (aa 1-125) was prepared as previously described [37]. Human myelin derived MBP was commercially purchased (Chemicon).

In Western-blots either $15 \mu \mathrm{g}$ recombinant MOG or $78 \mu \mathrm{g}$ MBP were loaded and separated in 10\% BisTris (Nupage) sodium dodecyl sulfate (SDS)-polyacrylamide gels (Novex, San Diego). Separated proteins were electrotransferred to nitrocellulose membranes (Hybond-C, Amersham). Efficiency of transfer was monitored by the use of prestained low range SDS-PAGE standard (Novex, SeeBlue Plus2) and by staining the membranes with Ponceau S (Sigma, St. Louis) after transfer.

Blots were blocked with $2 \%$ milk powder in phosphate buffered saline (PBS) containing $0.05 \%$ Tween-20 (PBS-T) for one hour, then dried, cut into $2 \mathrm{~mm}$ nitrocellulose strips and incubated overnight at $4^{\circ} \mathrm{C}$ with diluted human sera (1:500 in $2 \%$ milk powder in PBS$\mathrm{T})$. The strips were then washed three times with PBS-T and incubated with alkaline phosphatase conjugated anti-human IgM (1:5000, Jackson) for 1 hour at room temperature. After washing bound antibodies were detected by nitroblue tetrazolium chloride and 5-bromo-4-chloro-3-indolyl phosphate (Roche Molecular Diagnostics). The strips were then washed with distilled water, dried, and then assessed independently by two investigators (R.L.; F.H.) who had no access to clinical and MRI findings. A serum sample was considered to be "positive" if the immunoreactivity was greater than that of a standard "negative" control sample. Monoclonal antibodies to MBP (MAB381, Chemicon), and MOG (8.18-C5) [7], and positive and negative human serum samples determined earlier were used as controls.

\section{Routine CSF examination}

Cells were counted by phase contrast microscopy within one hour after lumbar puncture. The reference limit was 4.0 cells/ $\mu$ l. Albumin and IgG in CSF and corresponding serum were measured by automated immunoprecipitation nephelometry and the CSF/serum albumin ratio and IgG index $\left(\operatorname{IgG}_{\mathrm{CSF}} / \mathrm{IgG}_{\mathrm{Serum}}\right)$ :(albumin $\mathrm{CSF}_{\mathrm{CS}} / \mathrm{albu}-$ $\min _{\text {Serum }}$ ) calculated. Values $>0.7$ reflect intrathecal IgG synthesis [27]. The fraction (\%) of intrathecally produced IgG was calculated according to Reiber [36]. Oligoclonal IgG bands (OCB) were detected by agarose isoelectric focusing combined with immunoblotting and avidin-biotin amplified double-antibody peroxidase staining [20]. At least two sole OCB had to be present in CSF without corresponding bands in serum [2].

\section{Statistical evaluation}

Comparisons between groups according to antibody status were performed with the chi-square, Mann-Whitney U-test or Fisher's exact test as appropriate. A two-tailed $\mathrm{p}<0.05$ was considered statistically significant. The cumulative incidence of the development of CDMS was calculated for the groups with Kaplan-Meier estimates, and differences between the groups were evaluated with the log-rank test. Incidence rates were compared by chi-square test. The Cox proportional hazard model was used for univariate and multivariate analysis, to estimate relative risks according to antibody status (hazard ratios and 95 percent confidence intervals (CI)) with adjustment for potential confounding variables.

\section{Results}

39 sera were analyzed independently in Innsbruck (M. Reindl and T. Berger) and in our laboratory using identical methods to evaluate interlaboratory reproducibility. Strips were rated as "positive", and "negative" for anti-MOG- and anti-MBP antibodies. We obtained concordant results in $88.5 \%$ of the antiMOG- and $85.2 \%$ of the anti-MBP-determinations.

We analysed 133 sera overall from patients with CIS for anti-MOG and anti-MBP. The mean interval from initial symptoms to blood draw was 119 days (median 45 days, interquartile range: 106.5 days). The mean age was 34.9 years (SD: 10.1, range: $13-62$ ) and the gender distribution was $73.7 \% / 26.3 \%$ (f/m). Symptoms occurring from spinal lesions were more frequently observed than through involvement of brainstem or optic tracts $(37.6 \%$ vs. $18.0 \%$ and $18.8 \%$ ). In $21.1 \%$ there was clinical evidence for involvement of more than one anatomical region and in $4.5 \%$ the initial symptom was not specified.

$39.1 \%$ of the patients were single positive for antiMOG $(n=52)$, and $4.5 \%$ of the patients single positive for anti-MBP $(n=6) .30 .1 \%$ were anti-MOG and antiMBP double positive $(\mathrm{n}=40)$ and $26.3 \%$ had no antiMOG or anti-MBP (double negative group, $\mathrm{n}=35$ ).

Women were overrepresented in the group that scored positive for one or more antibodies: In the anti-MBP or anti-MOG positive groups, $89.1 \%$ and $78.3 \%$ respectively were females, as compared with $65.5 \%$ and $63.4 \%$ in the anti-MBP or anti-MOG negative groups $(p=0.003$ and 0.073$)$. In the double positive group, $87.5 \%$ were women, as compared with $57.1 \%$ in the double negative group $(\mathrm{p}=0.003)$. Other baseline variables like age, interval to blood draw, type of initial symptoms and clinical state of disease (relapse versus stable disease) did not relate to antibody status (Table 1 ).

$30 / 128(23.4 \%)$ patients lacked OCB and the frequency of OCB did not relate to antibody status. By contrast, quantitative CSF measures of inflammation were increased in the antibody positive patients (intrathecal IgG fraction: double negative group: $12.3 \%$; double positive group: $28.3 \%, \mathrm{p}=0.048$; $\mathrm{CSF}$ leukocyte count (median): double negative group: 3.0 cells $/ \mu \mathrm{l}$; double positive group: 7.0 cells $/ \mu \mathrm{l}, \mathrm{p}=0.036$ ). All patients had normal blood brain barrier function as measured by age-adjusted albumin ratio values (Table 2).

Since lesion load in MRI is associated with a higher risk of conversion to MS we evaluated signs of inflammation in the initial MRI in relation to antibody status. For 109 of 133 patients baseline MRIs were available. Patients with anti-MOG and anti-MBP showed more white matter lesions than patients 
Table 1 Baseline characteristics of the patients with CIS according to antibody status (groups B and C, Figure 1)

\begin{tabular}{|c|c|c|c|c|c|}
\hline & All patients & Anti-MOG - and Anti-MBP - & Anti-MOG + & Anti-MBP + & Anti-MOG + and Anti-MBP + \\
\hline Patients, n (\%) & 133 & $35(26.3)$ & $92(69.2)$ & $46(34.6)$ & $40(30.1)$ \\
\hline Females, n (\%) & $98(73.7)$ & $20(57.1)$ & $72(78.3)^{\mathrm{a}}$ & $41(89.1)^{\mathrm{a}}$ & $35(87.5)^{b}$ \\
\hline Age, years* & $34.9 \pm 10.1$ & $35.1 \pm 12.5$ & $34.3 \pm 8.9$ & $34.8 \pm 8.9$ & $33.7 \pm 8.3$ \\
\hline Optic neuritis, n (\%) & $25(18.8)$ & $4(11.4)$ & $18(19.6)$ & $10(21.7)$ & $7(18.9)$ \\
\hline Brainstem, n (\%) & $24(18.0)$ & $4(11.4)$ & 19 (20.7) & $11(23.9)$ & $10(25.0)$ \\
\hline Spinal, n (\%) & $50(37.6)$ & $18(51.4)$ & $31(33.7)$ & $12(26.1)$ & $11(27.5)$ \\
\hline$>1$ region**, n (\%) & $28(21.1)$ & $8(22.9)$ & $19(20.7)$ & $10(21.7)$ & $9(22.5)$ \\
\hline Relapse, n (\%) & $45(33.8)$ & $10(28.6)$ & $33(35.9)$ & $16(34.8)$ & $14(35.0)$ \\
\hline Interval*, days & $119 \pm 186$ & $97 \pm 157$ & $121 \pm 190$ & $116 \pm 150$ & $116 \pm 150$ \\
\hline
\end{tabular}

* Values are means \pm standard deviation. Significant findings are marked in bold.

** >1 anatomical region (multifocal first manifestation) [43].

${ }^{a} p=0.073$ and 0.003 for comparison with anti-MOG -/anti-MBP $-;{ }^{b} p=0.003$ for comparison with anti-MOG - and anti-MBP -

Table 2 Cerebrospinal fluid findings according to antibody status (groups B and C, Figure 1)

\begin{tabular}{|c|c|c|c|c|c|}
\hline & All patients & Anti-MOG - and Anti-MBP - & Anti-MOG + & Anti-MBP + & Anti-MOG + and Anti-MBP + \\
\hline Albumin ratio $(n=70)^{*}$ & $5.2 \pm 1.7$ & $5.4 \pm 1.8$ & $5.0 \pm 1.6$ & $5.3 \pm 1.6$ & $5.2 \pm 1.5$ \\
\hline $\operatorname{lgG}-\operatorname{Index}(n=70)^{*}$ & $0.98 \pm 0.58$ & $0.82 \pm 0.56$ & $1.03 \pm 0.59$ & $1.14 \pm 0.70$ & $1.16 \pm 0.72^{\mathrm{a}}$ \\
\hline Intrath. IgG $(n=70)(\%)^{*}$ & $21.6 \pm 25.0$ & $12.3 \pm 23.3$ & $24.2 \pm 25.1^{\mathrm{b}}$ & $27.2 \pm 27.6$ & $28.3 \pm 27.7^{c}$ \\
\hline Intrath. IgM $(n=70)(\%)^{*}$ & $2.6 \pm 10.5$ & $0.9 \pm 3.2$ & $3.2 \pm 11.9$ & $2.3 \pm 10.2$ & $2.6 \pm 10.7$ \\
\hline Intrath. $\lg A(n=70)(\%)^{*}$ & $2.0 \pm 8.7$ & $0.0 \pm 0.0$ & $2.7 \pm 9.9$ & $1.3 \pm 4.9$ & $1.4 \pm 5.2$ \\
\hline Leuc./ $\mu \mathrm{l}(\mathrm{n}=114)^{* *}$ & $4.3 \pm 10.5$ & $3.0 \pm 10.3$ & $6.0 \pm 10.9$ & $7.0 \pm 5.4^{d}$ & $7.0 \pm 5.5^{\mathrm{e}}$ \\
\hline OCB pos. $(n=128)(\%)$ & 76.6 & 78.8 & 75.6 & 80.0 & 80.0 \\
\hline
\end{tabular}

* Values are means \pm standard deviation. ${ }^{* *}$ Values are medians \pm standard deviation. Significant findings are marked in bold.

${ }^{a} p=0.051$ for comparison with anti-MOG - and anti-MBP $-;{ }^{b} p=0.056$ for comparison with anti-MOG $-;^{c} p=0.048$ for comparison with anti-MOG - and antiMBP -; ${ }^{d} p=0.023$ for comparison with anti-MBP -; ${ }^{e} p=0.036$ for comparison with anti-MOG - and anti-MBP -

without $(\mathrm{p}=0.01$, Figure $2 \mathrm{a})$. This difference results from higher lesion numbers in the anti-MBP positive versus negative patients $(p=0.007$, Figure $2 b)$, whereas such a correlation was not significant for anti-MOG alone $(p=0.166$, Figure $2 c)$. Accordingly, there was a trend towards lesion dissemination in space being more pronounced in patients with antiMBP than in patients with anti-MOG (anti-MBP: $67.5 \%$ vs. $50.0 \%$ fulfilled Barkhof criteria, $p=0.076$; anti-MOG: $60.0 \%$ vs. $48.5 \%, \mathrm{p}=0.266)$. Moreover, anti-MBP positive patients had more infratentorial lesions than the remainder of patients $(41.0 \%$ vs. $22.1 \%, p=0.037)$. We saw no additional antibody specific pattern of lesion distribution or preferential lesion localization according to antibody status.

Clinical follow-up data was available from $55 \mathrm{pa}-$ tients (10 patients without anti-MOG or anti-MBP, 43 patients with anti-MOG, 26 patients with anti-MBP and 24 patients with anti-MOG and anti-MBP). Transition to CDMS occurred in 58\% (32/55) of patients (mean duration: 14.6 months; SD: 9.7 months; range: $1-39$ months), whereas 23 patients did not experience a second clinical episode (mean follow-up 32.9 months, SD: 19.9 months, range 12-77.2 months).
Conversion to CDMS occurred more frequently in patients with anti-MOG versus no anti-MOG $(62.8 \%$ vs. $41.7 \%)$, and anti-MBP versus no anti-MBP $(69.2 \%$ vs. $48.3 \%$ ) and in patients positive for both antibodies compared with those without any ( $75 \%$ vs. $50 \%)$ but the differences did not reach significance $(p>0.1$ for each of these comparisons). Only in female patients with at least one T2 lesion did the difference between antibody status and conversion to CDMS reach significance for anti-MOG ( $\mathrm{n}=34$ ) (anti-MOG: $66.7 \%$ vs. $14.3 \%, \mathrm{p}=0.028$; anti-MBP: $66.7 \%$ vs. $43.8 \%, \mathrm{p}=$ 0.3 ; double positive: $75.0 \%$ vs. $20.0 \%, \mathrm{p}=0.047$ ) (Table 3).

The Kaplan Meier analysis shows a trend towards shorter time to second events in patients with at least one inflammatory lesion and anti-MOG antibodies (anti-MOG: 25.7 months, CI: 17.1-34.2; anti-MOG negative: 47.8 months, CI: 26.2-69.3, $\mathrm{p}=0.1062$, Figure $3 \mathrm{~b}$ ). In anti-MBP and double positive patients this was even less significant (anti-MBP: 26.8 months, CI: $16.5-37.0$, anti-MBP negative: 37.4 months, CI: 22.7-52.2, $\mathrm{p}=0.4886$; double positive: 21.7 months, CI: 13.7-29.8, double negative group: 39.6 months, CI:15.8-63.4, $\mathrm{p}=0.2102$ ) and for anti-MOG with regard to the entire follow-up group (anti-MOG: 30.3 

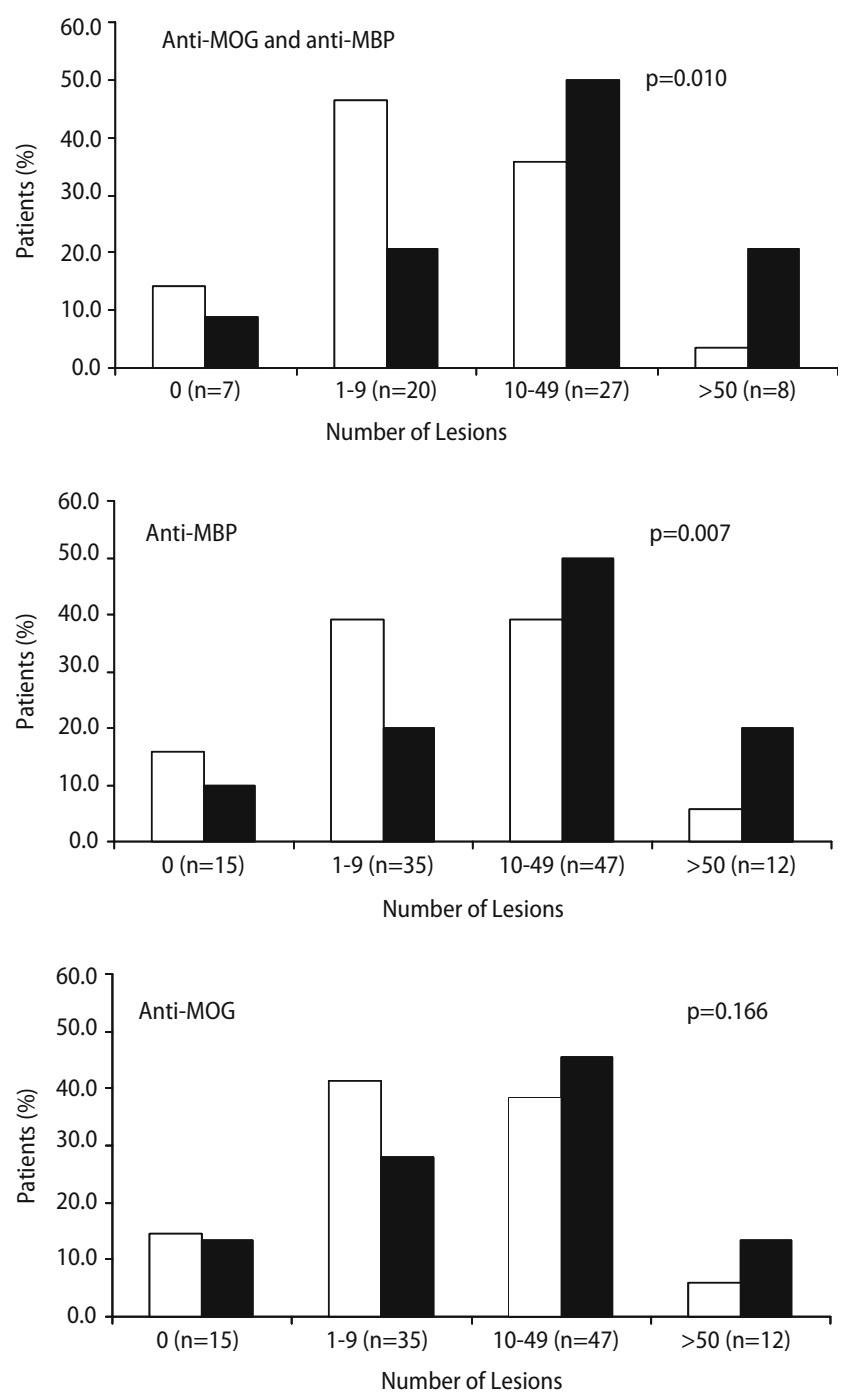

Fig. 2 Number of T2 hyperintense lesions according to antibody status for anti-MOG and anti-MBP- $(a ; n=62)$, anti-MBP- $(b ; n=109)$ and anti-MOG- $(c ;$ $n=109$ ) negative and positive patients. Open bars denote AB-negativity, black bars denote AB-positivity

months, CI: 21.8-38.8, anti-MOG negative: 44.3 months, CI: 23.9-64.7, $\mathrm{p}=0.2801$, Figure 3a). Again only in female patients with at least one T2 hyperintense lesion and anti-MOG or both antibodies did CDMS develop significantly faster (anti-MOG: 27.5 months, CI: 17.5-37.4; anti-MOG negative: 64.5 months, CI: 44.1-84.8, $\mathrm{p}=0.0209$, Figure 3c; double positive: 23.3 months, CI: $13.3-33.4$, double negative: 58.1 months, CI:31.6-84.6, $\mathrm{p}=0.0464)$. In the antiMBP positive group this was not visible (anti-MBP: 29.2 months, CI: 17.1-41.3; anti-MBP negative: 42.6 months, CI: 25.1-60.0, $\mathrm{p}=0.2868$ ).

The univariate Cox model confirmed that antiMOG and anti-MBP were not individual predictors of CDMS in the entire patient group ( $p=0.288$ and $p=0.359$ ). Again, female patients with at least one inflammatory lesion and anti-MOG showed a trend for increased risk of developing CDMS $(p=0.051$, risk ratio: 7.5 , CI: $0.1-57.0, \mathrm{n}=34$ ). In patients with both antibodies this trend was also visible ( $\mathrm{p}=$ 0.080 , risk ratio: 6.4 , CI: $0.8-51.4$ ) but not in patients with anti-MBP alone $(\mathrm{p}=0.296$, risk ratio 1.7 , CI: $0.6-4.3)$.

The multivariate model included the initial symptoms, relapse or stable disease status at the timepoint of blood drawing, the interval between first occurrence of symptoms and blood drawing, presence of OCB and CSF-leukocyte count. In females with at least one inflammatory lesion seropositivity for anti-MOG but not anti-MBP was associated with a 28.5 fold risk of CDMS compared with anti-MOG negative patients $(\mathrm{p}=0.005$, risk ratio $28.5, \mathrm{CI}: 2.7-300.4$; anti-MBP: $\mathrm{p}$ $=0.119$, risk ratio 2.5 , CI: $0.8-7.9$ ). The same multivariate approach failed to show any significant prognostic value of anti-MOG or anti-MBP positivity in the overall patient group $(n=55)$ (anti-MOG: $\mathrm{p}=$ 0.148 , risk ratio 2.1 , CI: $0.8-5.8$; anti-MBP: $p=0.133$, risk ratio 1.8 , CI: $0.8-3.8$ ).

\section{Discussion}

There are no well established, reliable markers to predict conversion of CIS to CDMS or to define subsets of patients for potential early treatment. In a previous study by Berger and colleagues, antibodies against MOG and MBP in CIS patients were highly predictive for CDMS. We aimed to confirm these findings with samples of an independent CIS patient cohort. We used Western blot technique to determine IgM antibodies against the extracellular, non-glycosylated MOG and purified human MBP used previously [4]. Our previous interlaboratory analysis (Innsbruck vs. Basel) showed sufficient reproducibility of this technique.

The initial clinical presentation of MS affects disease course and prognosis without allowing reliable individual long and especially short term predictions. CIS patients with optic neuritis, isolated sensory symptoms, long interval to second relapse and low disability tend to have a better long-term prognosis $[10,12,22,45]$.

Besides the fact that these clinical characteristics allow limited long-term predictions, MRI has also proven to be useful in estimating the interval to a second relapse and consequently CDMS. We found significantly more $\mathrm{T} 2$ hyperintense lesions in antiMBP alone or anti-MOG and anti-MBP positive patients compared to the other patient groups. In a prospective study $72 \%$ of the 57 patients with 
Table 3 Characteristics of patients with follow-up (group B, Figure 1)

\begin{tabular}{|c|c|c|c|c|c|}
\hline & All patients & Anti-MOG - and Anti-MBP - & Anti-MOG + & Anti-MBP + & Anti-MOG + and Anti-MBP + \\
\hline Patients, n (\%) & 55 & $10(18.2)$ & $43(78.2)$ & $26(47.3)$ & $24(43.6)$ \\
\hline Females, n (\%) & $43(78.2)$ & $6(60.0)$ & $35(81.4)$ & $23(88.5)$ & $21(87.5)$ \\
\hline Time to diagnosis (mths) & 14.6 & 15.6 & 14.4 & 15.3 & 15.3 \\
\hline Duration of follow-up (mths) & 32.9 & 30.5 & 32.1 & 41.0 & 39.4 \\
\hline Patients with CDMS, n (\%) & $32(58.2)$ & $5(50.0)$ & $27(62.8)$ & $18(69.2)$ & $18(75.0)$ \\
\hline - one T2 lesion, $n(\%)^{b}$ & $27(60.0)$ & $4(44.4)$ & $23(67.6)^{d}$ & $15(71.4)$ & $15(78.9)^{\mathrm{e}}$ \\
\hline - females, one T2 lesion, $\mathrm{n}(\%)^{\mathrm{c}}$ & $19(55.9)$ & $1(20.0)$ & $18(66.7)^{f}$ & $12(66.7)$ & $12(75.0)^{h}$ \\
\hline
\end{tabular}

* Values are means. Significant findings are marked in bold. ${ }^{a}$ Duration of follow-up in patients without CDMS. ${ }^{\mathrm{b}}$ Patients with at least one T2 lesion in the baseline MRI $(n=45)$. ' females with at least one T2 lesion in the baseline MRI $(n=34)$

${ }^{d} p=0.086$ for comparison with anti-MOG $-(36.4 \%) ;{ }^{e} p=0.097$ for comparison with anti-MOG - and anti-MBP -

f $p=0.028$ for comparison with anti-MOG - (14.3\%); ${ }^{\mathrm{h}} \mathrm{p}=0.047$ for comparison with anti-MOG - and anti-MBP -

abnormal MRI (single asymptomatic lesion at baseline) developed CDMS during a 5 year follow-up as compared with $6 \%$ in the group of patients without MRI lesions [31]. After 14 years 88\% with an abnormal baseline MRI developed CDMS whereas $19 \%$ with normal baseline MRI had a second clinical event over the same period [6]. In a cohort of 200 patients $49 \%$ of patients with either four or more T2-weighted lesions or three lesions, one of which had to be periventricular, developed CDMS within 2 years, compared with $5 \%$ of patients who had a normal baseline MRI [24]. In another study $71 \%$ of 60 CIS patients with three or more white matter lesions (at least one periventricular) progressed to CDMS after a mean follow-up of 14.3 months compared to only $24 \%$ of those without lesions [39].

In our study the frequency of OCB was not related to the myelin antibody status, but the amount of intrathecal IgG production and the CSF leukocyte counts were significantly higher in the anti-MOG and antiMBP positive patients. In previous studies increased intrathecal IgG concentrations and plasma cells were associated with a more active disease course [13]. In a group of 45 untreated patients with acute monosymptomatic optic neuritis a significant relation was found between leukocyte count and MRI as well as for IgGindex and MRI lesions, but not between results of MRI and presence of OCB [14]. An increased ratio between $B$ cells and monocytes in CSF was indicative of a pronounced disease progression in patients with relapsing remitting MS [8]. The specificity of the augmented intrathecal $B$ cell response in more active disease courses is unclear and it is still doubtful if this relates to intrathecally enriched anti-myelin antibody secreting cells $[33,42]$. Our findings of increased intrathecal IgG production and increased leukocyte counts in the antiMOG and anti-MBP positive patients are in line with previous reports on the role of humoral factors in the progression of multiple sclerosis.

There have been several studies focusing on disease specificity of anti-MOG rather than on prognostic value in CIS patients. Although the pathogenic role of the anti-MOG antibody response is well established in animal models and in vitro, there is still no evidence in MS $[18,21,26]$. The anti-MOG antibody response is unlikely to be a specific feature of MS, since it also has been detected in acute viral and bacterial CNS diseases. In the latter cases the antibody response seems to be transient, in contrast to its persistence in MS [37].

Lampasona and colleagues reported anti-MOG IgM antibodies at a very low frequency in patients with MS, encephalomyelitis and healthy control subjects (8\%) with no differences between groups using a liquidphase radiobinding assay (RIA). The antigen they used was full-length, non-glycosylated MOG (amino acids 1-218) [23]. Gaertner et al. used an ELISA technique with full-length, glycosylated mouse MOG and found elevated levels of antibodies in serum in CIS and in clinically active disease compared with healthy controls [15]. However, no other inflammatory, neurological diseases were studied. For assay specificity the glycosylation state of proteins, which affects conformation must be taken into account, since glycosylated proteins preserve their tertiary structure better than unglycosylated ones [40]. In the assay we used, as opposed to liquid-phase RIA, the antigen is immobilized on a membrane. We can not exclude loss of epitopes due to protein denaturation or exposure of new epitopes not present in the native conformation [32]. Liquid phase assays, in which a protein more likely retains its original conformation, in contrast to Western blot and ELISA techniques, might be an approach to study conformation dependent antibodies and their possible disease specificity $[5,23]$.

Lim and colleagues recently investigated the prognostic role of anti-MOG and anti-MBP in a cohort of 47 patients with CIS. Antibodies were analyzed by applying Western blot as described by Berger et al. [4]. It was noteworthy that 46 of the patients presented with optic neuritis and one patient had spinal cord involvement. In this study baseline antibody status did neither relate to the development of CDMS 

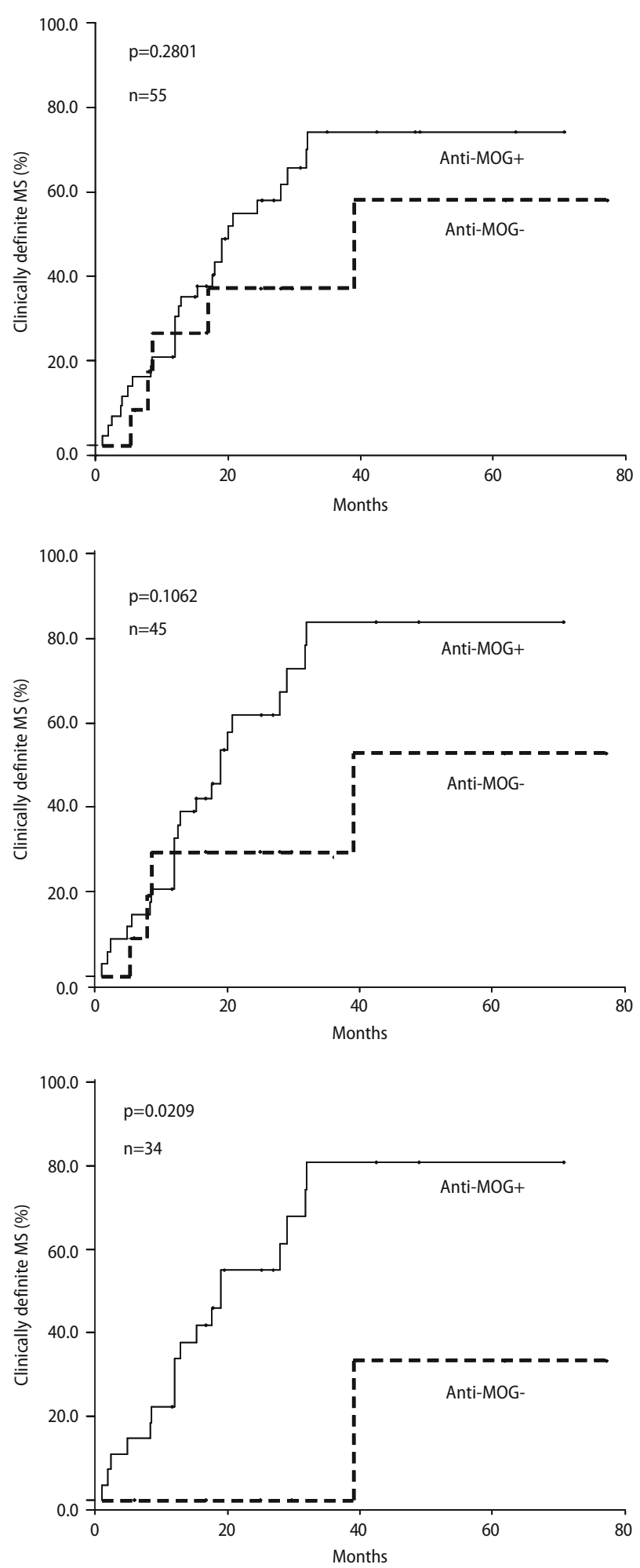

Fig. 3 Cumulative probability of clinically definite multiple sclerosis (CDMS) according to presence of anti-MOG antibodies in all patients with follow up $(a, n=55)$, patients with at least one T2 lesion in MRI at baseline $(b, n=45)$ and females with at least one T2 lesion in MRI at baseline $(c, n=34)$ after a follow-up of one year, nor was there any association with MRI findings at baseline or after follow-up [25].

In our study patients with anti-MOG and antiMBP developed CDMS more frequently $(75 \%)$ than patients without these antibodies (50\%), without reaching statistical significance. In a secondary exploratory analysis approach this was more pronounced in females with at least one T2 lesion. Berger et al. exlusively included patients who had at least two subclinical MRI lesions and OCB in the CSF. Since we quantified lesions per slice to consider spatial extension of lesions instead of real lesions it is difficult to compare the amount of abnormality in MRI between these two studies. Exclusion of patients without OCB from our followup group $(\mathrm{n}=11)$ did not yield an increased association between anti-MOG or anti-MBP and CDMS (data not shown). Importantly the gender distribution in the previous study by Berger et al. was similar to our follow-up group $(70.9 \%$ vs. $78.2 \%$ respectively) excluding a major gender driven effect. In the study by Berger et al serum samples for antibody analysis were collected within two weeks after the onset of the initial neurologic symptom [4]. Although in our patients we have not found differences in the risk of MS related to the interval of first symptoms to blood draw (data not shown), the delay of $119 \pm 186$ days in our study might have had an impact on the results. Although our study is not definitive due to the relatively low number of patients involved (33/55 patients converted to CDMS) and the limited duration of follow-up (mean 32.9 months), it supports the view that the prognostic value of anti-MOG and anti MBP antibodies is at best lower than initially indicated [4]. Studies with longer and systematic clinical and MRI followup in larger cohorts are needed to define more exactly the role of anti-myelin antibodies in predicting conversion to CDMS in CIS in general, or in well characterized patient subgroups.

Acknowledgements The authors thank Andy Schötzau, MD for statistical advice, Andrea Coors for technical assistance and Stacy $\mathrm{Wu}$ for MRI analysis. This work was supported by the Swiss MS Society and by an unrestricted grant from Schering Schweiz AG.

Disclosure J. Kuhle, R. L. P. Lindberg, A. Regeniter, M. Mehling, F. Hoffmann and M. Reindl have reported no conflicts of interest. E.W. Radue has received grant support from various pharmaceutical companies developing and marketing drugs in MS. D. Leppert is an employee of GlaxoSmithKline R \& D. T. Berger and L. Kappos have received grant support, consultancy fees and speaker honoraria from various pharmaceutical companies developing and marketing drugs for MS, exclusively for research support. 


\section{Reference}

1. Achiron A, Barak Y (2000) Multiple sclerosis-from probable to definite diagnosis: a 7-year prospective study. Arch Neurol 57:974-979

2. Andersson $M$, varez-Cermeno $J$, Bernardi G, Cogato I, Fredman P, Frederiksen J, Fredrikson S, Gallo P, Grimaldi LM, Gronning M (1994) Cerebrospinal fluid in the diagnosis of multiple sclerosis: a consensus report. J Neurol Neurosurg Psychiatry 57:897902

3. Baumann N, Pham-Dinh D (2001) Biology of Oligodendrocyte and Myelin in the Mammalian Central Nervous System. Physiol Rev 81:871-927

4. Berger T, Rubner P, Schautzer F, Egg $\mathrm{R}$, Ulmer $\mathrm{H}$, Mayringer I, Dilitz E, Deisenhammer F, Reindl M (2003) Antimyelin antibodies as a predictor of clinically definite multiple sclerosis after a first demyelinating event. $\mathrm{N}$ Engl J Med 349:139-145

5. Bonifacio E, Lampasona V, Bingley PJ (1998) IA-2 (islet cell antigen 512) is the primary target of humoral autoimmunity against type 1 diabetesassociated tyrosine phosphatase autoantigens. J Immunol 161:2648-2654

6. Brex PA, Ciccarelli O, O'Riordan JI, Sailer M, Thompson AJ, Miller DH (2002) A longitudinal study of abnormalities on MRI and disability from multiple sclerosis. N Engl J Med 346:158-164

7. Brunner C, Lassmann $\mathrm{H}$, Waehneldt TV, Matthieu JM, Linington C (1989) Differential ultrastructural localization of myelin basic protein, myelin/oligodendroglial glycoprotein, and $2^{\prime}, 3^{\prime}-$ cyclic nucleotide $3^{\prime}$-phosphodiesterase in the CNS of adult rats. J Neurochem 52:296-304

8. Cepok S, Jacobsen M, Schock S, Omer B, Jaekel S, Boddeker I, Oertel WH, Sommer N, Hemmer B (2001) Patterns of cerebrospinal fluid pathology correlate with disease progression in multiple sclerosis. Brain 124:21692176

9. Comi G, Filippi M, Barkhof F, Durelli L, Edan G, Fernandez O, Hartung H, Seeldrayers P, Sorensen PS, Rovaris M, Martinelli V, Hommes OR (2001) Effect of early interferon treatment on conversion to definite multiple sclerosis: a randomised study. Lancet 357:1576-1582

10. Confavreux C, Vukusic S, Adeleine P (2003) Early clinical predictors and progression of irreversible disability in multiple sclerosis: an amnesic process. Brain 126:770-782
11. Egg R, Reindl M, Deisenhammer F, Linington C, Berger T (2001) AntiMOG and anti-MBP antibody subclasses in multiple sclerosis. Mult Scler 7:285-289

12. Eriksson M, Andersen O, Runmarker B (2003) Long-term follow up of patients with clinically isolated syndromes, relapsing-remitting and secondary progressive multiple sclerosis. Mult Scler 9:260-274

13. Farlow MR, Edwards MK, Kolar OJ, Stevens JC, Yu PL (1987) Magnetic resonance imaging in multiple sclerosis: analysis of correlations to peripheral blood and spinal fluid abnormalities. Neurology 37:15271530

14. Frederiksen JL, Larsson HB, Olesen J (1992) Correlation of magnetic resonance imaging and CSF findings in patients with acute monosymptomatic optic neuritis. Acta Neurol Scand 86:317-322

15. Gaertner S, de Graaf KL, Greve B, Weissert R (2004) Antibodies against glycosylated native MOG are elevated in patients with multiple sclerosis. Neurology 63:2381-2383

16. Galboiz Y, Miller A (2002) Immunological indicators of disease activity and prognosis in multiple sclerosis. Curr Opin Neurol 15:233-237

17. Genain CP, Cannella B, Hauser SL, Raine CS (1999) Identification of autoantibodies associated with myelin damage in multiple sclerosis. Nat Med 5:170-175

18. Genain CP, Nguyen $\mathrm{MH}$, Letvin $\mathrm{NL}$, Pearl R, Davis RL, Adelman M, Lees MB, Linington C, Hauser SL (1995) Antibody facilitation of multiple sclerosis-like lesions in a nonhuman primate. J Clin Invest 96:2966-2974

19. Jacobs LD, Beck RW, Simon JH, Kinkel RP, Brownscheidle CM, Murray TJ, Simonian NA, Slasor PJ, Sandrock AW (2000) Intramuscular interferon beta1a therapy initiated during a first demyelinating event in multiple sclerosis. CHAMPS Study Group. N Engl J Med 343:898-904

19a. Kappos L, Polman CH, Freedman MS, Edan G, Hartung HP, Miller DH, Montalban X, Barkhof F, Bauer L, Jakobs P, Pohl C, Sandbrink R, for the BENEFIT Study Group (2006) Treatment with interferon beta 1-6 delays conversion to clinically definite and McDonald Ms in patients with clinically isolated syndromes. Neurology 67:1242-1249
20. Keir G, Luxton RW, Thompson EJ (1990) Isoelectric focusing of cerebrospinal fluid immunoglobulin G: an annotated update. Ann Clin Biochem 27 (Pt 5):436-443

21. Kerlero de RN, Honegger P, Lassmann H, Matthieu JM (1990) Demyelination induced in aggregating brain cell cultures by a monoclonal antibody against myelin/oligodendrocyte glycoprotein. J Neurochem 55:583-587

22. Kurtzke JF, Beebe GW, Nagler B, Kurland LT, Auth TL (1977) Studies on the natural history of multiple sclerosis-8. Early prognostic features of the later course of the illness. J Chronic Dis 30:819-830

23. Lampasona V, Franciotta D, Furlan R, Zanaboni S, Fazio R, Bonifacio E, Comi G, Martino G (2004) Similar low frequency of anti-MOG IgG and IgM in MS patients and healthy subjects. Neurology 62:2092-2094

24. Lee KH, Hashimoto SA, Hooge JP, Kastrukoff LF, Oger JJ, Li DK, Paty DW (1991) Magnetic resonance imaging of the head in the diagnosis of multiple sclerosis: a prospective 2-year follow-up with comparison of clinical evaluation, evoked potentials, oligoclonal banding, and CT. Neurology 41:657-660

25. Lim ET, Berger T, Reindl M, Dalton CM, Fernando K, Keir G, Thompson EJ, Miller DH, Giovannoni G (2005) Anti-myelin antibodies do not allow earlier diagnosis of multiple sclerosis. Multi Scler 11:492-494

26. Linington $C$, Bradl $M$, Lassmann $H$, Brunner C, Vass K (1988) Augmentation of demyelination in rat acute allergic encephalomyelitis by circulating mouse monoclonal antibodies directed against a myelin/oligodendrocyte glycoprotein. Am J Pathol 130:443-454

27. Link H, Tibbling G (1977) Principles of albumin and IgG analyses in neurological disorders. III. Evaluation of IgG synthesis within the central nervous system in multiple sclerosis. Scand J Clin Lab Invest 37:397-401

28. Linnington $\mathrm{C}$, Webb $\mathrm{M}$, Woodhams PL (1984) A novel myelin-associated glycoprotein defined by a mouse monoclonal antibody. J Neuroimmunol 6:387-396

29. Lucchinetti CF, Bruck W, Rodriguez M, Lassmann H (1996) Distinct patterns of multiple sclerosis pathology indicates heterogeneity on pathogenesis. Brain Pathol 6:259-274 
30. Miller DH, Ormerod IE, Gibson A, du Boulay EP, Rudge P, McDonald WI (1987) MR brain scanning in patients with vasculitis: differentiation from multiple sclerosis. Neuroradiology 29:226-231

31. Morrissey SP, Miller DH, Kendall BE, Kingsley DP, Kelly MA, Francis DA, MacManus DG, McDonald WI (1993) The significance of brain magnetic resonance imaging abnormalities at presentation with clinically isolated syndromes suggestive of multiple sclerosis. A 5-year follow-up study. Brain 116 (Pt 1):135-146

32. O'Connor KC, Chitnis T, Griffin DE, Piyasirisilp S, Bar-Or A, Khoury S, Wucherpfennig KW, Hafler DA (2003) Myelin basic protein-reactive autoantibodies in the serum and cerebrospinal fluid of multiple sclerosis patients are characterized by low-affinity interactions. J Neuroimmunol 136:140-14833

33. Olsson $\mathrm{T}$ (1994) Multiple sclerosis:cerebrospinal fluid. Ann Neurol 36 Suppl:S100-S102

34. Ormerod IE, Bronstein A, Rudge P, Johnson G, Macmanus D, Halliday AM, Barratt H, Du Boulay EP, Kendal BE, Moseley IF, . (1986) Magnetic resonance imaging in clinically isolated lesions of the brain stem. J Neurol Neurosurg Psychiatry 49:737743

35. Pagany M, Jagodic M, Schubart A, Pham-Dinh D, Bachelin C, Baron van EA, Lachapelle F, Olsson T, Linington C (2003) Myelin oligodendrocyte glycoprotein is expressed in the peripheral nervous system of rodents and primates. Neurosci Lett 350:165-168
36. Reiber H (1994) Flow rate of cerebrospinal fluid (CSF)-a concept common to normal blood-CSF barrier function and to dysfunction in neurological diseases [see comments]. J Neurol Sci 122:189-203

37. Reindl M, Linington C, Brehm U, Egg R, Dilitz E, Deisenhammer F, Poewe W, Berger T (1999) Antibodies against the myelin oligodendrocyte glycoprotein and the myelin basic protein in multiple sclerosis and other neurological diseases: a comparative study. Brain 122 (Pt 11):2047-2056

38. Schluesener HJ, Sobel RA, Linington C, Weiner HL (1987) A monoclonal antibody against a myelin oligodendrocyte glycoprotein induces relapses and demyelination in central nervous system autoimmune disease. J Immunol 139:4016-4021

39. Sharief MK, Thompson EJ (1991) The predictive value of intrathecal immunoglobulin synthesis and magnetic resonance imaging in acute isolated syndromes for subsequent development of multiple sclerosis. Ann Neurol 29:147-151

40. Stefferl A, Brehm U, Linington C (2000) The myelin oligodendrocyte glycoprotein (MOG): a model for antibody-mediated demyelination in experimental autoimmune encephalomyelitis and multiple sclerosis. J Neural Transm Suppl 123-133
41. Storch MK, Stefferl A, Brehm U, Weissert R, Wallstrom E, Kerschensteiner $\mathrm{M}$, Olsson T, Linington C, Lassmann H (1998) Autoimmunity to myelin oligodendrocyte glycoprotein in rats mimics the spectrum of multiple sclerosis pathology. Brain Pathol 8:681-694

42. Sun J, Link $H$, Olsson $T$, Xiao BG, Andersson G, Ekre HP, Linington C, Diener P (1991) T and B cell responses to myelin-oligodendrocyte glycoprotein in multiple sclerosis. J Immunol 146:1490-1495

43. Uitdehaag BM, Kappos L, Bauer L, Freedman MS, Miller D, Sandbrink R, Polman C (2005) Discrepancies in the interpretation of clinical symptoms and signs in the diagnosis of multiple sclerosis. A proposal for standardization. Multi Scler 11:227-231

44. Villoslada P, Abel K, Heald N, Goertsches R, Hauser SL, Genain CP (2001) Frequency, heterogeneity and encephalitogenicity of $\mathrm{T}$ cells specific for myelin oligodendrocyte glycoprotein in naive outbred primates. Eur J Immunol 31:2942-2950

45. Weinshenker BG, Bass B, Rice GP, Noseworthy J, Carriere W, Baskerville J, Ebers GC (1989) The natural history of multiple sclerosis: a geographically based study. 2. Predictive value of the early clinical course. Brain $112(\mathrm{Pt}$ 6):1419-1428 